\title{
Personal benefits from post-ingestional actions of dietary constituents
}

\author{
BY D. A. BOOTH, J. A. FRENCH, C. J. WAINWRIGHT AND \\ A. J. H. GATHERER \\ Nutritional Psychology Research Group, School of Psychology, University of Birmingham, \\ Edgbaston, Birmingham B15 2TT
}

The study of cognitive effects of ingested dietary constituents remains at an almost alchemical stage. Much as in the search for the philosopher's stone, investigators of cognitive effects of foods stir a substance into a brew and then filter groups of people who have consumed the brew through an array of tests, looking for grains of 'gold' such as mood elevation, more effective intellectual or athletic performance, or signs of the prospect of a popular new drink or food product. Even the much studied substances alcohol and caffeine are still tested empirically, rather than investigated using theoretically well-founded conceptions of the mechanisms involved.

The difficulty in studying cognitive effects of dietary constituents lies not so much in the measurement of subjective experiences or behavioural performance, as in the inadequacy of the theoretical basis for such studies. Psychological measurement technology developed over the past 50 years or more compares favourably with current techniques for, say, dietary measurement. Cognitive psychologists and exercise scientists can readily construct sound and practical measurements, for example, of ability to concentrate or remember, or of cardiovascular fitness. Also, the answers to questions about emotional state or beliefs about competence can be shown by multi-variate psychometric criteria to be reliable quantitative expressions of underlying cognitive determinants: indeed, mood scales are better validated than many food sensory scoring systems.

\section{NEUROSCIENCE AND COGNITION}

Concepts of how food substrates might affect behaviour by acting on the brain are crude at best. Heavy reliance is placed on textbook myths about the roles in behaviour of different brain regions and neurotransmitters (Booth \& Stribling, 1983; Booth, 1990). The psychoactivity of compounds in foods or drinks or of other natural substances that might be used is generally considered in terms only of known pharmacology. Since serotonin, adenosine and $\gamma$-aminobutyric acid synapses, for example, and their and other receptor subtypes are spread over multitudinous neuronal circuits in many parts of the brain, knowing that a food constituent may act on a particular receptor class has few, if any, implications for the constituent's effects on psychologically specific processes engineered in the brain. Even a highly receptor-specific psychiatric drug can have complex effects on mood and cognitive and muscular performance.

Thus, from what has long been known of brain structure and processing, it is most unlikely that a food constituent will prove to affect mood, attention or memory specifically, let alone any single process within such broad cognitive functions. To hope that it might is to misconceive the relationship between neurophysiology and psychology.

The psychoactive user. It follows from such considerations that food constituents are liable to have diffuse cognitive effects. It is also likely that the effects of widely used 
constituents are much weaker than those, for example, of purpose-synthesized moodaltering agents. Hence, the psychological results of the action of food constituents on the brain may depend greatly on the context in which they occur and how they relate to the activities of the user. Indeed, the specificity and power of a constituent's cognitive effects are liable to derive from functional benefits to ongoing experience or performance that the user has consciously or unconsciously learned to exploit. The user may be more psychoactive than the food constituent in the effects produced.

Such learned use of a neural effect is not merely an expectancy effect. A placebo would evoke that. The psychological effect depends on noticing some effect that the food substance actually has and taking it as evidence that the expected effect is occurring; as a result, the incipient emotional reaction gains conviction and the incentive increases to apply oneself in that manner to the tasks in hand. For instance, if a 'cup of cheer' produces a warm feeling under the diaphragm, that sensation can be cheering in itself and make it easier to smile at someone else. Such autosuggestive processes, therefore, can build on diffuse and weak physical effects to produce specific and strong behavioural patterns.

Existing effects. This may be particularly relevant for product developers who, rather than starting with substances known to have pharmacological activity, may find it highly productive to measure existing or desired uses of foods and drinks and to seek to identify substances in them that consumers have already found to be cognitively useful. These uses may have been developed by quite small groups of people. The traditions that other cultures have developed from locally available plants, etc., within their own social practices could be a rich source of dietary benefits. Such traditional uses also have some safety assurance already attached. Therefore, a promising and ethical approach for those seeking items to promote for their psychological effects would be the experimental analysis of the ethnopharmacology of traditional tonics and the recognized cheering or relaxing drinks or foods.

For the same reasons, psychologists concerned with the basic mechanistic analysis of cognitive effects of diet could usefully focus on the benefits that consumers are already attributing to available products. Rational development in the pharmaceutical industry now generally derives from understanding of physiological processes involved in drug treatment. Central nervous system (CNS) pharmacology has to be an exception because it is concerned with psychiatric, neurological and autonomic disorders for which we may never have sufficiently specific central neurophysiology to design medication from first principles for the human brain. Only clinical tests are relevant to psychotherapeutic efficacy and so new CNS agents have to be screened by attempting to model the abnormal brain functions in animals. Fundamental research on food, mood and performance is under much less constraint technically and ethically. However, the only tests that are directly relevant to real-life situations are minor variants of what the individual consumer is already habitually doing.

To date, however, the experimental designs intended to investigate cognitive effects have imposed test foods and tasks on undifferentiated groups of people out of the context of the diverse wishes and expectations acquired from cultural conventions and personal experience. This deficiency in design is quite apart from the requirements for effective investigation arising also from genetic differences and individuals' varied levels of drug tolerance as a result of recent usage level (Dews, 1984). 
Mood and performance. A constituent's pattern of effects on performance in a battery of cognitive tests will not necessarily characterize what particular mental processes are affected and in what way. This requires a more analytical design of investigation, comparing tasks that are related in a way that identifies a change in the operation of a specific cognitive mechanism.

There is a variety of ways of identifying and measuring a cognitive process. The distinction between paper-and-pencil tests and computer-based tests is irrelevant provided the test reliably discerns true experimental differences and provided it yields interpretable evidence about the causal processes and how they have been affected.

The difference between measurements of so-called mood and so-called cognitive performance is often between people claiming to be able to do something and then demonstrating the ability. A mood rating is an assessment by the rater of a certain aspect of the rater's self-ascribed mental state, which may be manifest in emotional behaviour or in effects on cognitive performance. These different aspects of behaviour, measurable through performance tests (objectively) or self-ascription scores (subjectively), can interact with each other. For example, someone may feel more sociable because he or she is able to succeed in a task relevant to social esteem, or a person may perform a negotiation better because he or she is in a confident but relaxed state of mind. Thus, what is not generally recognized, even in psychology, and is relevant to data mentioned later in the present paper, is that expressions of emotional state are often also claims to cognitive efficiency (or admissions of inefficiency), which may be recognized in social interactions rather than intellectual tasks. Emotions are the subjective side of objective social roles (Coulter, 1979; Forgas, 1988). Verbal expressions of emotion, therefore, typically carry implications of social ability. If I say I am in a happy mood, I am expressing my subjective view, but $I$ am also making claims that may be assessed objectively, such as at present being able to smile easily and speak cheerfully to others. Thus, whether intellectual, social or emotional in character, both the solving of problems and making judgements about one's own state can be evidence of cognitive efficiency.

However, the psychologists of thought and perception (even when interested in emotion) have generally confined their research to intellectual tasks. Many social psychologists now call their approach cognitive, in the sense that they invoke thinking in their theoretical explanations of social perception and action (Eiser, 1986). Nevertheless, as yet few measures of cognitive performance have been introduced into sociopsychological investigation (Wegener, 1980). While the measurement of emotional state by facial expression is now highly developed (Ekman, 1984), the vastly richer and more abundant social information provided by words still remains without criterion-validated performance measures.

\section{INDIVIDUAL SPECIFICITY OF CAFFEINE EFFECTS}

Caffeine drinks are widely used in Western society to help maintain wakefulness and to aid concentration in a mentally demanding task. Yet this may not have been the emphasis when tea and coffee were first introduced to Britain. Furthermore, it is not the emphasis laid by advertisers of cola drinks. They appear to find it profitable to associate the brands with physically energetic activity, social interaction or having fun, or both. (Without seeing market research data and the creative briefs, however, the possibility must be allowed that advertisers are merely cultivating a common self-image among 
heavy purchasers.) Tea also may be widely regarded in Britain as the cup that cheers quite as much as it refreshes. (Again, though, it may be possible to distinguish sociable use from sociable mood.)

Given such diverse or diffuse roles for caffeine-containing drinks, the investigation of the effects of caffeine on cognitive efficiency must not be set too exclusively in an intellectual mould. Also, users may differ in their expectations of a cup of coffee, depending on the range of situations in which they have enjoyed the drink and perhaps actually benefited from it psychologically. In other words, the mood and performance effects for someone who drinks tea mainly while a host or guest at tea parties may be different from those for someone who uses it to revive flagging energy during the afternoon at the office. People who drink tea mainly at breaks at work may gain both sorts of benefit. Indeed, it may be difficult to distinguish the two types of mood and of performance in such people unless investigations include different occasions of drink consumption and consider a variety of effects.

Elevations of social moods have been reported in group experiments on caffeine, but they have been interpreted as secondary to felt improvements on the intellectual tasks set for the caffeine-treated participants (Lieberman et al. 1987). Our initial studies have not compelled participants to face challenges to their intellectual abilities and so any social mood effects observed are unlikely to be open to this interpretation.

Frequent users of caffeinated tea or coffee volunteered to record for 1 week their feelings in their own words while getting a drink and 10-15 min after finishing it. Coded stocks were provided of three versions of teabags or instant coffee powder of the respondent's usual type or as similar as practicable. The three versions were prepared from a commercially decaffeinated variety of the drink material, by adding caffeine to the level in the individual's usual material, adding twice that amount or applying a blank treatment that gave the material the same appearance as the recaffeinated versions. After the first test day using their habitual tea or coffee, participants used each of the three codes as a replacement for their normal drink for two non-consecutive days in the test week. Individual participants drank between five and twelve cups at each level of caffeine.

An initial interview elicited situations and states of mind which the participant associated with their drinks. Participants were each given a booklet in which to record and rate their state before and after each drink. The state was described by the participant's own word or phrase and rated on a line $100 \mathrm{~mm}$ long, with the terms 'not at all' at one end and 'extremely' at the other end. Mood change (item) was estimated by subtracting predrink from post-drink scores. Predrink states and mood changes were then subjected separately to principal components analysis to obtain factor scores.

Of the first two dozen individuals whose data were analysed, only one showed no significant variation in predrink or mood-change scores over the caffeine levels or over different types of drink. Two-fifths of these participants showed significant variation between caffeine levels in at least one mood-factor. Data from illustrative individuals are given as values including all statistically significant factor and item scores $(P<0.05)$ from that person. The factors are described by the words for the items that were most heavily loaded on the factor, in the order listed. The person in Fig. 1 appears to show a 'pick-meup' effect with coffee containing double her usual amount of caffeine. The participant's feeling of being 'run down' was reduced after the drink; this term, possibly used for lowered alertness, was correlated with feeling sociable in this person. 

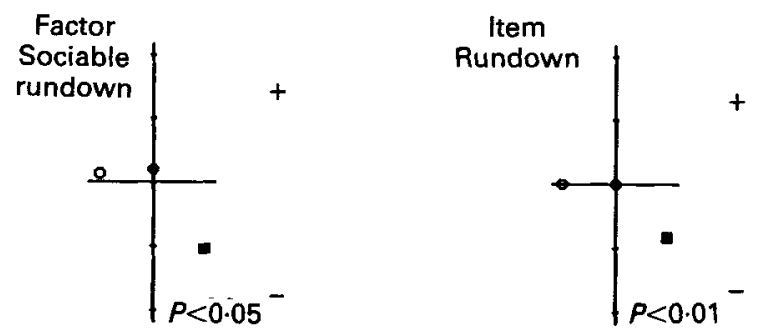

Fig. 1. Factor or item score differences for self-ascribed mood of participant no. 530 from before to after coffee drinks containing $7(\mathrm{O}), 80(\mathrm{O})$ and $160(\mathrm{D}) \mathrm{mg}$ caffeine in $200 \mathrm{ml}$. The vertical scales are marked at intervals of $10 \%$ above and below the individual's predrink state.

(a)

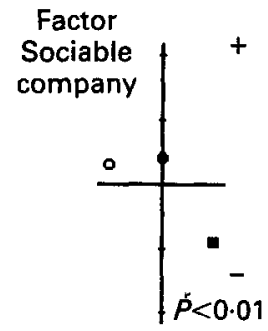

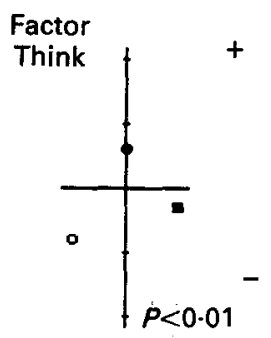

(b)
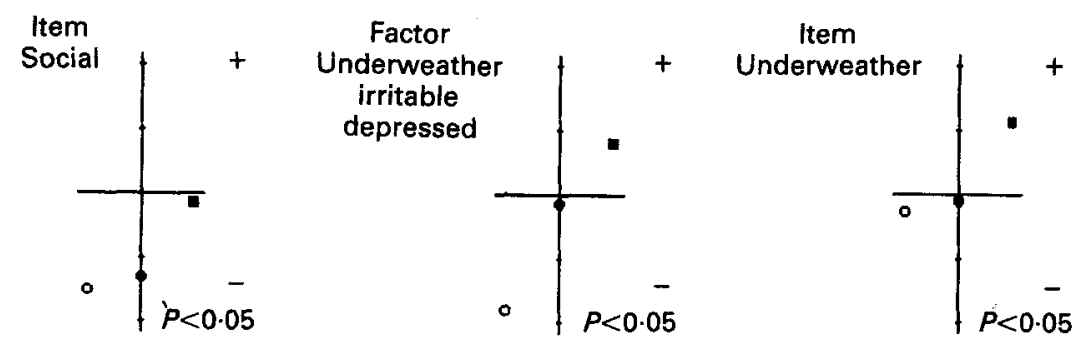

Fig. 2. (a) Self-ascription factor scores of participant no. 070 when preparing his usual drink of coffee containing $6(\mathrm{O}), 60(\mathbf{O})$ or $120(\mathbb{0}) \mathrm{mg}$ caffeine in $200 \mathrm{ml}$, (b) with factor or item score differences from before to after the drink.

Another participant (Fig. 2), like several others, appeared to experience some aversive effects from doubling the usual amount of caffeine in his coffee. He felt more 'under the weather' after the double-caffeine drinks. Indeed the general low mood on which this item loaded most heavily was reduced by decaffeinated coffee, relative even to the normal caffeine level. This may explain the paradoxical tendency to take coffee when needing a drink to 'help think'. A positive effect of caffeine can, however, be seen in a decrease in becoming less 'social'. This effect may account for the tendency to take the double-caffeinated coffee when not feeling 'sociable' or in need of 'company'. Since the dosing of the drink materials was disguised, a predrink effect must be a learned anticipation of the effects of the drink over the two test days. Hence, we have evidence of 
Table 1. Effects of a tryptophan-free amino-acid-rich tonic on users in benefit-relevant tasks

(Frequencies among fifteen subjects)

\begin{tabular}{lccc}
\hline & \multicolumn{2}{c}{ Frequency of effect on performance } \\
\cline { 3 - 4 } Task & Improvement & Worsening & No effect \\
\hline Letter cancellation: & 8 & 2 & 5 \\
$\quad$ Accuracy & 6 & 2 & 7 \\
Speed & 5 & 2 & 8 \\
Mental arithmetic & & & 10 \\
Verbal recall: & 3 & 2 & 10 \\
Immediate & 4 & 1 & \\
Delayed & & & \\
\hline
\end{tabular}

the reinforcing effects of the drinks on subsequent choices, as well as the effects at the time of consumption. Moreover, despite participants being asked to substitute their test drinks for their usual one in a predetermined way, they were, it seems, still able to decide when to take that drink in the light of their current state.

\section{CULTURAL SPECIFICITY OF TRYPTOPHAN DEPLETION}

Because the cognitive effects of food constituents depend on the history of use of available materials, traditional tonics are liable to be culture-specific. These potions or delicacies are held in high regard by the cultures that use them for a variety of benefits to a sense of well-being. It was noted that students from one ethnic group consumed a commercially well-established version of a traditional tonic during revision and examination periods. This preparation was rich in gelatin, a protein that contains effectively no tryptophan.

Such users may feel some counter-sedative effect of a very slight reduction in serotoninergic activity (Young et al. 1985; Spring et al. 1987). This might be induced by raised plasma levels of large neutral amino acids other than tryptophan if the tonic is consumed sufficiently long after a meal. The slight increase in felt arousal or alertness would be what is expected and needed. So it might encourage greater effort and closer attention to the intellectual task in hand. Thus, autosuggestive processes could amplify and focus a small and non-specific effect into a substantial and discrete effect on mental concentration and protracted attention.

This understanding of cognitive effects of foods has been supported in preliminary tests of the tonic carried out in this University. A quantitative simulation of proofreading (striking out four target letters in lines of random letters) provided a test of sustained attention; improvements in accuracy of letter cancellation appeared to be more frequent than benefits to the other tasks tested in a group of users of the tonic (Table 1). However, no such signs of an effect on vigilance have thus far been seen with this dose of the tonic under these normal conditions in groups from a culture where this tonic and this use of it are effectively unknown.

The possibility arises that the glucose tablets and drinks used during examinations and sporting activities, and indeed caffeine drinks (and tablets), may similarly have at least 
part of their effects by such a culturally acquired autosuggestive process. The real effects on cognitive and athletic efficiency, and the mechanisms by which they arise from personal uses of neural actions, will only be identified by analysis of systematic patterns in individuals' behaviour in familiar contexts.

This work was supported in part by a grant from the Agricultural and Food Research Council and the British Nutrition Foundation.

\section{REFERENCES}

Booth, D. A. (1990). The behavioral and neural sciences of ingestion. In Neurobiology of Food and Fluid Intake, pp. $465-488$ [E. M. Stricker, editor]. New York: Plenum Press.

Booth, D. A. \& Stribling, D. (1978). Neurochemistry of appetite mechanisms. Proceedings of the Nutrition Society 37, 181-191.

Coulter, J. (1979). Social Construction of Mind. London: Macmillan.

Dews, P. B. (1984). Behavioral effects of caffeine. In Caffeine, pp. 86-103 [P. B. Dews, editor]. New York: Springer-Verlag.

Eiser, J. R. (1986). Social Psychology, Attitudes, Cognition \& Social Behaviour. Cambridge: Cambridge University Press.

Ekman, P. (1984). Expression and nature of emotion. In Approaches to Emotion, pp. 319-343 [K. S. Scherer and P. Ekman, editors]. Hillsdale, N.J.: Erlbaum, Lawrence, Associates Inc.

Forgas, J. P. (1988). Affect in social and personal judgments. In Affect, Cognition and Social Behaviour [K. Fiedler and J. P. Forgas, editors]. Toronto: Horgrefe.

Lieberman, H. R., Wurtman, R. J., Garfield, G. S., Roberts, C. H. \& Coviella, I. L. (1987). The effects of low doses of caffeine on human performance and mood. Psychopharmacology 92, 308-312.

Spring, B., Chiodo, J. \& Bowen, D. J. (1987). Carbohydrates, tryptophan and behavior: a methodological review. Psychological Bulletin 102, 234-256.

Wegener, B. (editor) (1980). In Social Attitudes and Psychophysical Measurement. Hillsdale, N.J.: Erlbaum, Lawrence, Associates, Inc.

Young, S. N., Smith, S., Pihl, R. O. \& Ervin, F. R. (1985). Tryptophan depletion causes a rapid lowering of mood in normal males. Psychopharmacology 87, 173-177. 